\title{
Detection of saliva-range glucose concentrations using organic thin-film transistors
}

\author{
D. Elkington, W. J. Belcher, P. C. Dastoor, and X. J. Zhou \\ Centre for Organic Electronics, University of Newcastle, Callaghan, New South Wales 2308, Australia
}

(Received 10 May 2014; accepted 22 July 2014; published online 30 July 2014)

\begin{abstract}
We describe the development of a glucose sensor through direct incorporation of an enzyme (glucose oxidase) into the gate of an organic thin film transistor (OTFT). We show that glucose diffusion is the key determinant of the device response time and present a mechanism of glucose sensing in these devices that involves protonic doping of the transistor channel via enzymatic oxidation of glucose. The integrated OTFT sensor is sensitive across 4 decades of glucose concentration; a range that encompasses both the blood and salivary glucose concentration levels. As such, this work acts as a proof-of-concept for low-cost printed biosensors for salivary glucose. (C) 2014 AIP Publishing LLC. [http://dx.doi.org/10.1063/1.4892012]
\end{abstract}

Development in glucose sensing technology has increased rapidly in recent decades with the heightened incidence of diabetes worldwide. According to a recent report, by the year 2030 , the proportion of adult sufferers of diabetes in the world is predicted to be $7.7 \%$ of the population (or $438.7 \times 10^{6}$ people) compared with $6.4 \%$ in 2010 . $^{1}$ Currently, people with diabetes need to monitor their blood glucose levels multiple times daily in order to be able to determine appropriate times to eat and to administer medication. Existing sensing equipment used by diabetics requires a small sample of blood to be taken, which can be painful and inconvenient. ${ }^{2}$ As such, there is demand for a more userfriendly, non-invasive approach for measuring the concentration of glucose in blood. In addition, even within the area of existing blood glucose sensors, there is a need for increased sensitivity of the glucose concentration measurement. A recent study of commercial blood glucose sensors by Freckmann et al. has shown that many commercial blood glucose sensors suffer from poor accuracy in the high and low blood glucose concentration ranges, which in turn impairs clinical decision making. ${ }^{3}$

Several reports have already shown that there exists a direct relationship between blood glucose levels and the concentration of glucose in saliva, ${ }^{4-7}$ which is approximately $8-200 \mu \mathrm{M}^{7}$ This range of glucose concentration is much lower than the range of concentration for glucose in blood, which is typically $3.3-8.3 \mathrm{mM}^{8}{ }^{8}$ Thus, if current detection sensitivity can be improved, the measurement of saliva glucose levels offers a path towards reliable, non-invasive methods for glucose concentration monitoring in blood.

The detection of glucose has been the subject of much research in the field of organic electronics, and several varieties of sensors have been reported. ${ }^{9}$ Conventional electrochemistry techniques (such as cyclic voltammetry and amperometry) have been employed to determine the concentration of the glucose analyte using the enzyme glucose oxidase (GOX) and these studies have shown promise with very high sensitivities reported. ${ }^{10,11}$ More recently, work has focussed on developing methods for immobilising the GOX in a polymer matrix for use as the electrode in electrochemical cells. ${ }^{12-14}$ Early work by Macaya et al. showed that micromolar sensitivity to glucose could be achieved in organic thin film transistor (OTFT) devices based on the polymer blend, poly(3,4-ethylenedioxythiophene) doped with poly(styrene sulfonate) (PEDOT:PSS), as the semiconducting channel. ${ }^{15}$ This work was further expanded by Kanakamedala et al. who showed that these PEDOT:PSS electrochemical devices could be fabricated using an inexpensive and rapid xurography technique. ${ }^{16}$ However, all of these previous studies required the GOX to be added to the glucose analyte, which is impractical for a commercial sensor. To date, there have been no reports of an integrated OTFT glucose sensor where the GOX is immobilised within the device structure itself.

In this Letter, we outline the development of a simple OTFT design featuring poly(3-hexylthiophene) (P3HT) as the semiconducting layer, and a gate electrode comprising a film of Nafion (a polymeric membrane material) loaded with a sensing enzyme. This electrode forms the recognition element of the OTFT-based sensor and exhibits sensitivities to glucose in the sub-millimolar concentration range. The work presented here demonstrates a practical self-contained architecture for a printable OTFT-based glucose sensor that is sensitive in the range of concentrations for glucose in saliva, where the enzyme is embedded into the device itself.

Figures 1(a) and 1(b) show the OTFT architectures used for the control OTFT device and the glucose sensing device, respectively. Both architectures are based on source and drain indium tin oxide (ITO) electrodes patterned on a glass substrate. ${ }^{17}$ The main difference between the two
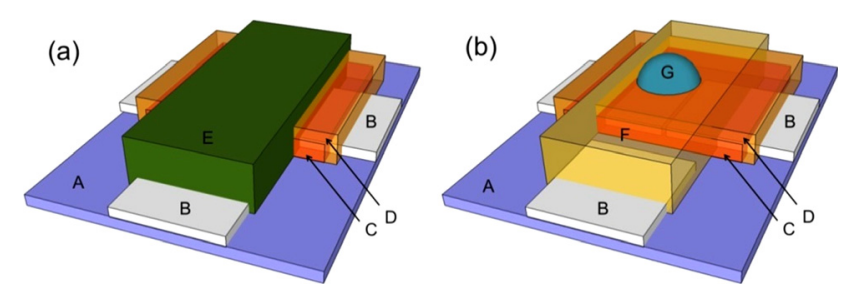

FIG. 1. Device configuration of two types of OTFT: (a) a "standard" device and (b) a glucose sensing OTFT featuring the enzyme GOX showing a droplet of analyte material. Materials used-A: glass, B: ITO, C: P3HT, D: PVP, E: PEDOT:PSS, F: Nafion:GOX, G: analyte. 
architectures is that the PEDOT:PSS gate electrode of the "standard" OTFT is replaced with the Nafion:GOX film. Atomic force microscopy (AFM) images of the devices were used to probe the surface morphology of each layer in the device. ${ }^{17}$ Whereas smooth films are obtained for the P3HT $\left(\mathrm{R}_{\mathrm{RMS}}=0.88 \mathrm{~nm}\right)$, polyvinylphenol (PVP) $\left(\mathrm{R}_{\mathrm{RMS}}\right.$ $=1.14 \mathrm{~nm}$ ), and Nafion $\left(\mathrm{R}_{\mathrm{RMS}}=5.20 \mathrm{~nm}\right)$, the Nafion $/ \mathrm{GOX}$ films exhibit micron height features on the surface, indicative of aggregates of the GOX enzyme embedded within the Nafion film.

The electrochemistry of the GOX-glucose interaction is well established. ${ }^{18}$ When GOX selectively oxidises glucose, gluconolactone, and hydrogen peroxide $\left(\mathrm{H}_{2} \mathrm{O}_{2}\right)$ are generated as products of the reaction as described in the following equation:

$$
\text { Glucose }+\mathrm{O}_{2} \stackrel{\text { GOX }}{\longrightarrow} \text { gluconolacetone }+\mathrm{H}_{2} \mathrm{O}_{2} \text {. }
$$

Subsequently, when the $\mathrm{H}_{2} \mathrm{O}_{2}$ in solution is oxidised above a threshold voltage $(\sim 0.7 \mathrm{~V}$ versus a standard calomel electrode), it breaks down electrochemically into protons, oxygen, and electrons as described in the following equation: ${ }^{19}$

$$
\mathrm{H}_{2} \mathrm{O}_{2} \stackrel{\sim 0.7 \mathrm{VvsSCE}}{\longrightarrow} \mathrm{O}_{2}+2 \mathrm{H}^{+}+2 e^{-} \text {. }
$$

The net result of these two reactions is that a stoichiometric quantity of protons is liberated which is directly proportional to the number of glucose molecules oxidised by the enzyme. In order to confirm the activity of GOX once, it is embedded in a Nafion film, films of Nafion:GOX were deposited onto ITO-coated glass and used as working electrodes in an electrochemical cell containing glucose in the electrolyte. Cyclic voltammetry data using these working electrodes showed that the production of ions from the electrochemical breakdown of $\mathrm{H}_{2} \mathrm{O}_{2}$ was proportional to the concentration of glucose molecules present in the system as expected. ${ }^{17}$

A key advantage of the OTFT architecture used here is its low voltage operation, which makes these devices wellsuited for operating with aqueous solutions, since water is known to undergo oxidation at voltages above $\sim 1.2 \mathrm{~V} .{ }^{20} \mathrm{In}$ order to determine the operating voltage window for oxidation of $\mathrm{H}_{2} \mathrm{O}_{2}$ in water, the amperometric response of Nafion:GOX films to aqueous $\mathrm{H}_{2} \mathrm{O}_{2}$ and a deionised (DI) water control was measured. In this configuration, the operating voltage window is defined as the voltage region between the onset of oxidation of the $\mathrm{H}_{2} \mathrm{O}_{2}$ and the onset of oxidation of water.

As can be seen in Figure 2(a), the onset of oxidation for $\mathrm{H}_{2} \mathrm{O}_{2}$ occurs at a voltage of $\sim 0.75 \mathrm{~V}$, whereas the onset of oxidation for water does not occur until $\mathrm{V} \approx 1.5 \mathrm{~V}$. Therefore, to avoid the oxidation of water contributing to changes in current in the sensing experiments, no electrical potential exceeding $1.5 \mathrm{~V}$ should be present in the device, whereas a voltage of at least $0.75 \mathrm{~V}$ is sufficient to oxidise (and generate charge carriers from) $\mathrm{H}_{2} \mathrm{O}_{2}$.

Figures 2(b) and 2(c) show the output characteristics corresponding to the two types of OTFT devices shown in Figure 1. The device with the PEDOT:PSS gate (Figure 2(b)) shows clearly defined linear and saturation regions as expected for these transistors. Furthermore, this device fully
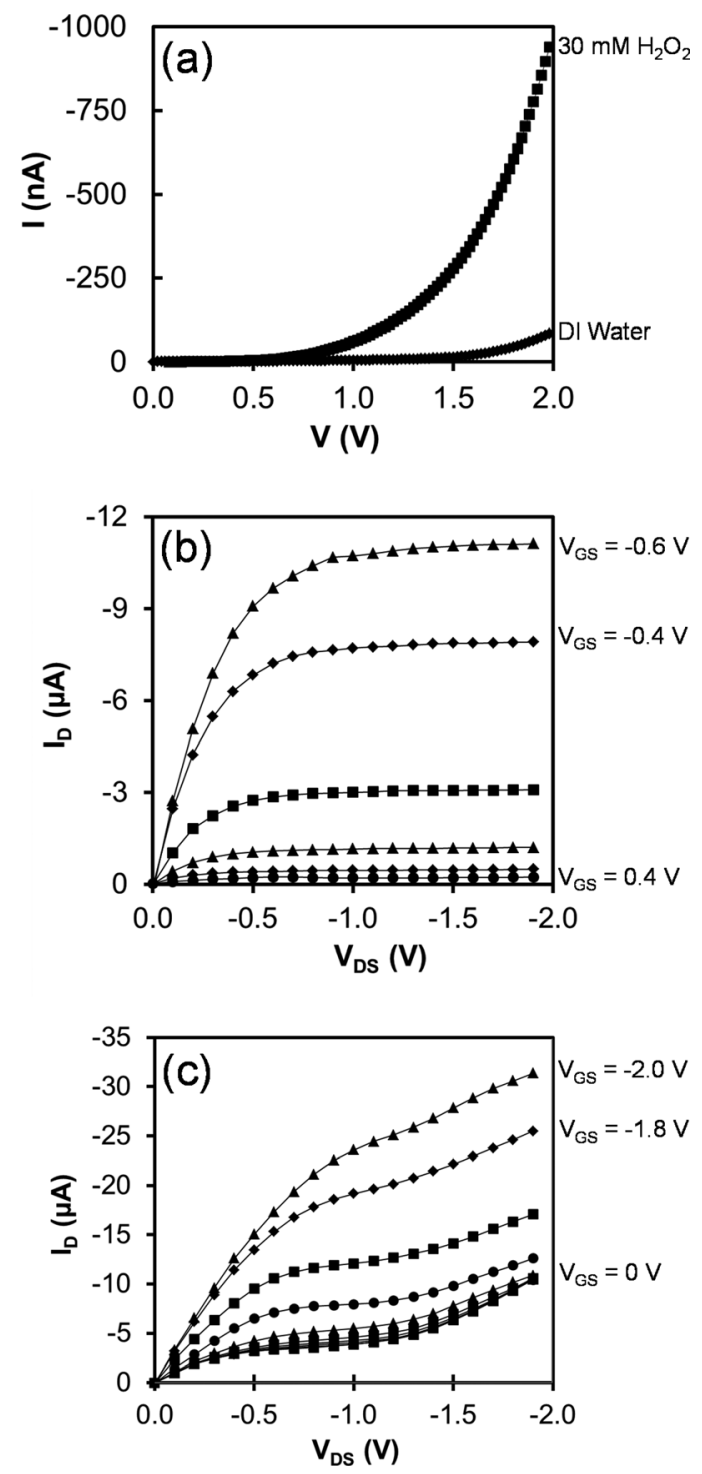

FIG. 2. (a) Current as a function of voltage between two ITO electrodes connected by a Nafion:GOX film to $10 \mu \mathrm{l}$ of both $30 \mathrm{mM} \mathrm{H}_{2} \mathrm{O}_{2}$ solution and DI water, (b) output characteristic of a P3HT/PVP/PEDOT:PSS OTFT, and (c) output characteristic of P3HT/PVP/Nafion:GOX OTFT devices.

turns on at a relatively low voltage of $\left|\mathrm{V}_{\mathrm{GS}}\right|<1 \mathrm{~V}$, which is characteristic of devices of this type. ${ }^{21-24}$ By comparison, the device with a Nafion:GOX layer on top of the PVP dielectric (Figure 2(c)) shows a less-clearly defined saturation region as well as relatively high drain current $\left(\mathrm{I}_{\mathrm{D}}\right)$ levels when the device is switched off. The deviation from saturation (linearly increasing $\mathrm{I}_{\mathrm{D}}$ versus $\mathrm{V}_{\mathrm{DS}}$ for $\mathrm{V}_{\mathrm{DS}}<\sim-1.1 \mathrm{~V}$ ) is consistent with a gate electrode offset from the sourcedrain channel. ${ }^{22}$ However, for operating source-drain voltages $0>\mathrm{V}_{\mathrm{DS}}>-1.0 \mathrm{~V}$, the low voltage transistor output characteristics are maintained when the PEDOT:PSS electrode is replaced with Nafion:GOX.

To measure the response of the P3HT/PVP/Nafion:GOX sensor devices to a range of glucose concentrations, a $10 \mu \mathrm{l}$ drop of analyte was placed on the Nafion:GOX electrode and the drain current $\left(I_{D}\right)$ was monitored as a function of time. ${ }^{17}$ $V_{D S}$ and $V_{G S}$ were set to $-1 \mathrm{~V}$ for the sensing experiments to ensure the OTFT sensor was operating: (i) above the threshold for charge generation from $\mathrm{H}_{2} \mathrm{O}_{2}$, (ii) below the 
threshold for oxidation of water, and (iii) within the relatively flat saturation region for P3HT/PVP/Nafion:GOX OTFT devices.

Figure 3 shows typical $\mathrm{I}_{\mathrm{D}}$ versus time measurements for glucose and $\mathrm{H}_{2} \mathrm{O}_{2}$ solutions as well as deionised water deposited onto the OTFT sensing devices. There is little change in OTFT device response due to the addition of deionised water, confirming that the applied voltages are below the threshold required to oxidise the solvent. Second, the addition of $\mathrm{H}_{2} \mathrm{O}_{2}$ results in an immediate and rapid increase in $\mathrm{I}_{\mathrm{D}}$, which reaches a plateau level of $\sim 30 \mu \mathrm{A}$, indicating that the applied voltage is above the threshold needed to breakdown $\mathrm{H}_{2} \mathrm{O}_{2}$ into protons. Finally, the addition of glucose produces a monotonic increase in $\mathrm{I}_{\mathrm{D}}$, which reaches a level $(\sim 25 \mu \mathrm{A})$ after 1200 seconds; similar to that of the $\mathrm{H}_{2} \mathrm{O}_{2}$ case.

We hypothesis that the difference in the temporal response between the $\mathrm{H}_{2} \mathrm{O}_{2}$ and glucose analytes arises from the slower diffusion and two-stage reaction of glucose with the embedded GOX enzyme compared with the direct diffusion and breakdown of $\mathrm{H}_{2} \mathrm{O}_{2}$ into protons. To test this hypothesis, we have fitted the $30 \mathrm{mM}$ glucose and $\mathrm{H}_{2} \mathrm{O}_{2}$ data to a one dimensional solution of Fick's second law of diffusion, $n(x, t)=n_{0} \operatorname{erfc}(x / 2 \sqrt{D t})=n_{0} \operatorname{erfc}(A / \sqrt{t})$, where $n_{0}$ (initial concentration), $x$ (diffusion distance), and $D$ (diffusion constant) are fitting constants. The excellent fit to the glucose and $\mathrm{H}_{2} \mathrm{O}_{2}$ data indicates that the variation of $\mathrm{I}_{D}$ arises from a diffusion process within the device. From the ratio of the glucose and $\mathrm{H}_{2} \mathrm{O}_{2}$ data fitting constants $\left(A_{G} / A_{P}=3.4\right)$, we calculate that the ratio of the effective diffusion constants for glucose and $\mathrm{H}_{2} \mathrm{O}_{2}\left(D_{P}^{\text {eff }} / D_{G}^{\text {eff }}\right)$ is 11.7, suggesting that the effective glucose diffusion is an order of magnitude slower than that of the $\mathrm{H}_{2} \mathrm{O}_{2}$.

The operation of these OTFT sensing devices is governed by the generation of charge carriers by the enzymatic reaction and their effect on OTFT current modulation. The current modulation in these hygroscopic OTFT devices is primarily mediated by the diffusion of protons through the dielectric layer. ${ }^{21-25}$ Indeed, we have shown that

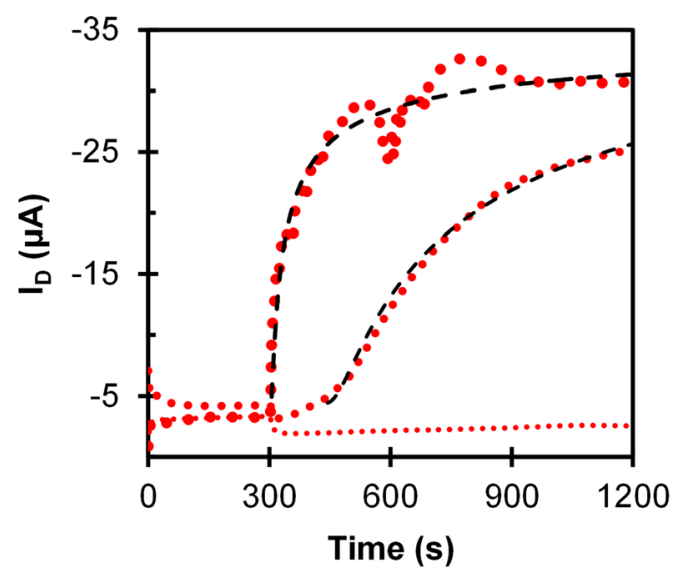

FIG. 3. Drain current as a function of time for OTFT sensing devices in the presence of water (small filled red circles), $30 \mathrm{mM}$ glucose (filled red circles), and $30 \mathrm{mM} \mathrm{H}_{2} \mathrm{O}_{2}$ (large filled red circles). The analyte was added at a time of $300 \mathrm{~s}$ in each case. The dashed lines correspond to fits to the $30 \mathrm{mM}$ glucose and $30 \mathrm{mM} \mathrm{H}_{2} \mathrm{O}_{2}$ data of the solution to the diffusion equation described in Eq. (3). incorporating a basic polyvinylpyridine (PVPy) dielectric layer leads to proton immobilisation, resulting in low device off-currents. ${ }^{25}$ Based on this previous work, we propose the following model for current generation in these OTFT glucose sensing devices (Figure 4). As discussed above, glucose diffusion through the PEDOT gate and Nafion to the GOX is slow and likely to be the rate determining step in the mechanism. By contrast, glucose is oxidised rapidly at the GOX to generate gluconolactone and $\mathrm{H}_{2} \mathrm{O}_{2},{ }^{26}$ which is subsequently rapidly oxidised to protons at the built-in device potential $(\sim 0.7 \mathrm{~V})$. Moreover, since PVP is a highly hydrated sol-gel material, any enzymatically generated protons will transfer rapidly to the PVP/P3HT interface via the Grotthuss mechanism ${ }^{27}$ whereupon they dope the P3HT semiconducting layer resulting in the observed $\mathrm{I}_{\mathrm{D}}$ changes.

Finally, the OTFT response to a range of glucose concentrations $(8 \mu \mathrm{M}-30 \mathrm{mM})$ was recorded. The dimensionless parameter $\chi$ was plotted as a function of glucose concentration where $\chi$ is given by

(a)

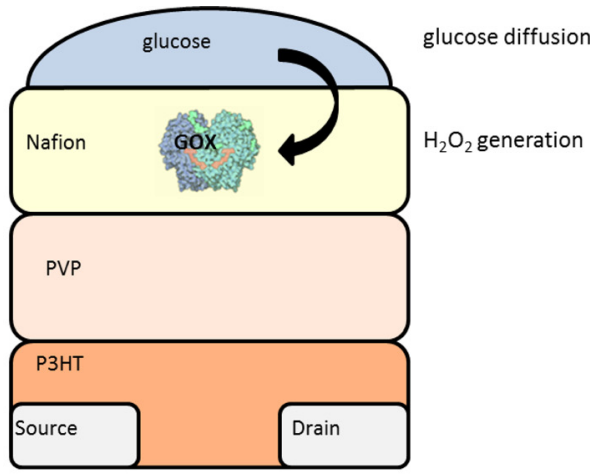

(b)

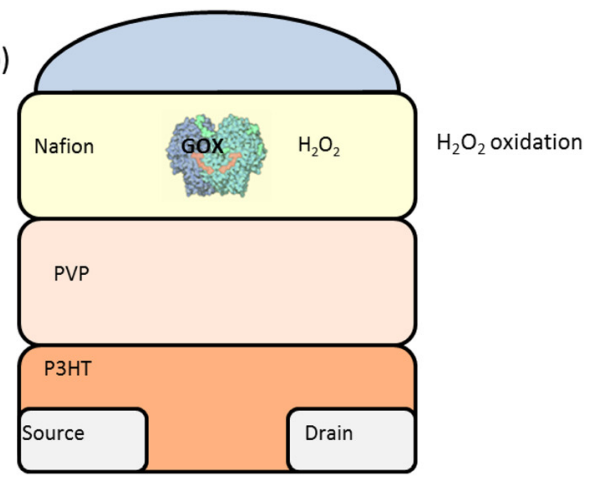

(c)

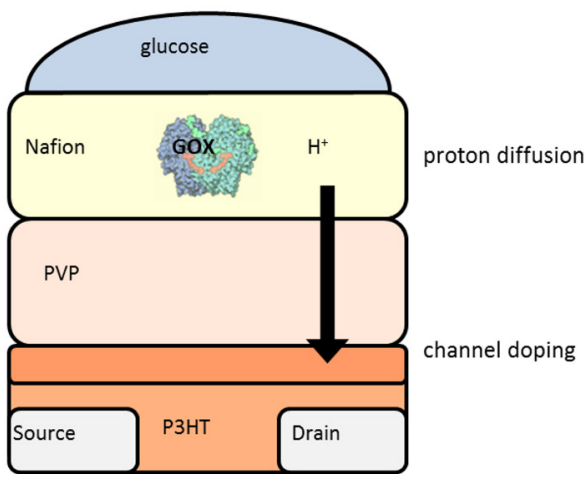

FIG. 4. Model for OTFT glucose sensing mechanism. A: Glucose diffuses to the GOX in the Nafion matrix whereupon $\mathrm{H}_{2} \mathrm{O}_{2}$ is generated. $\mathrm{B}$ : The $\mathrm{H}_{2} \mathrm{O}_{2}$ is oxidised and protons are generated. C: Protons then diffuse to and dope the P3HT channel. 


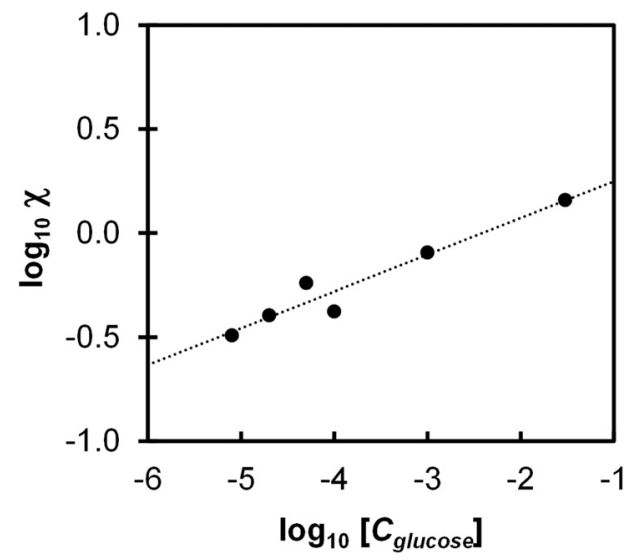

FIG. 5. Average normalised response $\left(\log _{10} \chi\right)$ for various glucose concentrations $(8 \mu \mathrm{M}, 20 \mu \mathrm{M}, 50 \mu \mathrm{M}, 100 \mu \mathrm{M}, 1 \mathrm{mM}$, and $30 \mathrm{mM})$ as a function of $\log _{10}\left[C_{\text {glucose }}\right]$. The calibration line is calculated to the data within standard error using least squares fitting. The error of the slope of the calibration line is $\pm 13 \%$.

$$
\chi=\frac{\int_{t=0}^{t=800}\left[I_{D}(t)-I_{D}(0)\right] d t}{\int_{t=0}^{t=800} I_{D}(0) d t} .
$$

The parameter $\chi$ is defined as the normalised area under the $\mathrm{I}_{\mathrm{D}}$ versus time response curve for $0 \leq \mathrm{t} \leq 800 \mathrm{~s}$. By considering the integral of the signal and normalising it in terms of the initial conductivity of the P3HT film, this dimensionless parameter accounts for any variation in the initial conductivity of the P3HT film or rate of change of current. Figure 5 shows a log-log plot of $\chi$ as a function of glucose concentration. We observe good correlation between the change in $I_{D}$ and glucose concentration over 4 decades of glucose concentration. Importantly, this range covers the concentrations observed both in saliva $(8-200 \mu \mathrm{M})$ and blood $(3-8 \mathrm{mM})$.

In summary, we have shown that GOX can be integrated into low-voltage OTFT devices without loss of enzyme activity or the need for chemical modification of the polymer matrix or enzyme. The integrated sensor shows a response across 4 decades of glucose concentration encompassing both saliva and blood glucose concentration levels. We propose a model for glucose sensing based on protonic doping of the transistor channel, which highlights that the diffusion of glucose is the key determinant of the device response time. This work acts as a proof-of-concept for the integration of functioning biomolecules (such as enzymes) directly into organic electronic devices and demonstrates the feasibility for low-cost printed biosensors for salivary glucose.

This work was performed in part at the Materials node of the Australian National Fabrication Facility, which is a company established under the National Collaborative Research Infrastructure Strategy to provide nano and microfabrication facilities for Australia's researchers. Ms. K. Sirois is gratefully acknowledged for cyclic voltammetry measurements. The authors also wish to acknowledge the contribution of an anonymous reviewer to the revised manuscript.

${ }^{1}$ J. E. Shaw, R. A. Sicree, and P. Z. Zimmet, Diabetes Res. Clin. Pract. 87, 4-14 (2009).

${ }^{2}$ W. H. Smart and K. Subramanian, Diabetes Technol. Ther. 2, 549-559 (2000).

${ }^{3}$ G. Freckmann, C. Schmid, A. Baumstark, S. Pleus, M. Link, and C. Haug, J. Diabetes Sci. Technol. 6, 1060-1075 (2012).

${ }^{4}$ S. Amer, M. Yousuf, P. Siddqiui, and J. Alam, Pak. J. Pharm. Sci. 14, 33-37 (2001).

${ }^{5}$ K. M. Karjalainen, M. L. Knuuttila, and M. L. Käär, Pediatr. Dent. 18, 306-311 (1996).

${ }^{6}$ M. Yamaguchi, Y. Kawabata, S. Kambe, F. Nystrom, K. Naitoh, and H. Yoshida, Med. Biol. Eng. Comput. 42, 322-327 (2004).

${ }^{7}$ M. Yamaguchi, M. Mitsumori, and Y. Kano, IEEE Eng. Med. Biol. Mag. 17, 59-63 (1998).

${ }^{8}$ R. T. Lagua and V. S. Claudio, Nutrition and Diet Therapy Reference Dictionary, 4th ed. (Chapman and Hall, 1996).

${ }^{9}$ C. Liao and F. Yan, Polym. Rev. 53, 352-406 (2013).

${ }^{10}$ C. Bartic, A. Campitelli, and S. Borghs, Appl. Phys. Lett. 82, 475-477 (2003).

${ }^{11}$ T. B. Singh, N. Marjanović, G. J. Matt, N. S. Sariciftci, R. Schwödiauer, and S. Bauer, Appl. Phys. Lett. 85, 5409 (2004).

${ }^{12}$ Y. Himuro, M. Takai, and K. Ishihara, Sens. Actuators, B 136,122-127 (2009).

${ }^{13}$ K. Khun, Z. H. Ibupoto, J. Lu, M. AlSalhi, M. Atif, A. A. Ansari, and M. Willander, Sens. Actuators, B 173, 698-703 (2012).

${ }^{14}$ M. Ammam and E. B. Easton, Sens. Actuators, B 155, 340-346 (2011).

${ }^{15}$ D. J. Macaya, M. Nikolou, S. Takamatsu, J. T. Mabeck, R. M. Owens, and G. G. Malliaras, Sens. Actuators, B 123, 374-378 (2007).

${ }^{16}$ S. K. Kanakamedala, H. T. Alshakhouri, M. Agarwal, and M. A. DeCoster, Sens. Actuators, B 157, 92-97 (2011).

${ }^{17}$ See supplementary material at http://dx.doi.org/10.1063/1.4892012 for experimental procedure, AFM data, and cyclic voltammetry data.

${ }^{18} \mathrm{P}$. Bartlett and R. Whitaker, J. Electroanal. Chem. Interfacial Electrochem. 224, 37-48 (1987).

${ }^{19}$ J. Liu, M. Agarwal, and K. Varahramyan, Sens. Actuators, B 135, 195-199 (2008).

${ }^{20} \mathrm{~W}$. M. Latimer, The Oxidation States of the Elements and Their Potentials in Aqueous Solutions, 2nd ed. (Prentice-Hall, New York, 1952).

${ }^{21}$ T. G. Bäcklund, H. G. O. Sandberg, R. Österbacka, and H. Stubb, Appl. Phys. Lett. 85, 3887 (2004).

${ }^{22}$ T. G. Bäcklund, R. Österbacka, H. Stubb, J. Bobacka, and A. Ivaska, J. Appl. Phys. 98, 074504 (2005).

${ }^{23}$ H. G. O. Sandberg, T. G. Bäcklund, R. Österbacka, S. Jussila, T. Mäkelä, and H. Stubb, Synth. Met. 155, 662-665 (2005).

${ }^{24}$ H. G. O. Sandberg, T. G. Bäcklund, R. Österbacka, and H. Stubb, Adv. Mater. 16, 1112-1115 (2004).

${ }^{25}$ D. Elkington, D. Darwis, X. Zhou, W. Belcher, and P. C. Dastoor, Org. Electron. 13, 153-158 (2012).

${ }^{26}$ Z. Tao, R. A. Raffel, A.-K. Souid, and J. Goodisman, Biophys. J. 96, 2977-2988 (2009).

${ }^{27}$ T. Li, G. Zhong, R. Fu, and Y. Yang, J. Membr. Sci. 354, 189-197 (2010). 